\title{
Signal Processing Approaches for Jitter Extraction in Time-of-Flight Range Imaging Cameras
}

\author{
Gehan Anthonys*, Michael J. Cree ${ }^{\dagger}$ and Lee Streeter ${ }^{\ddagger}$ \\ School of Engineering, University of Waikato, Hamilton 3240, New Zealand \\ Email: *gehan.anthonys@gmail.com, $\left\{{ }^{\dagger}\right.$ michael.cree, ${ }^{\dagger}$ lee.streeter $\} @$ waikato.ac.nz
}

\begin{abstract}
The precision and accuracy of time-of-flight fullfield range cameras are important for many applications, however there are a number of noise sources that degrade both precision and accuracy. Many of the noise sources such as nonlinearity, multipath inferences and harmonic cancellation are well investigated. Barely investigated is jitter on the camera light and shutter signals. Here we measure periodic and random jitter on the light signal of a camera. We use signal processing techniques to construct a reference signal, hence find the jitter. The performance of the proposed method is examined using the MESA Imaging SwissRanger 4000. We found periodic jitter of two frequencies at 0.12 and $5.04 \mathrm{MHz}$ and the random jitter of $164 \pm 4 \mathrm{ps}$, on the light signal of the camera.
\end{abstract}

\section{INTRODUCTION}

The technologies used to build range sensors have rapidly improved in camera manufacturing industries over the last couple of decades [1]-[3]. Time-of-fight (ToF) range imaging cameras are now commercially available at reasonable prices (i.e. a couple of hundred US dollars) and have high demand in many areas such as medical imaging [4], [5], computer graphics [6], mobile robotics [7], [8], 3D reconstruction [9], and human identification [10], [11] are some of them. ToF cameras measure the time taken for light to travel from the light source in the camera to the scene and back to the camera and it is this principle that is used in depth cameras to perform depth measurements. These measurements can be measured using either continuous wave (CW) modulation which is based on measuring phase differences between the emitted and received signals, or pulsed wave (PW) modulation which is based on measurement of the time of a light pulse trip to calculate the range image [12], [13].

Commercially available ToF cameras have relatively high noise levels due to electronic sources such as shot noise, reset noise, amplifier noise, crosstalk, analogue to digital converters (ADC) quantization and clock jitter, and due to multipath light interference. Many noise sources in these cameras such as harmonic contamination [14], [15], non-linearity, multipath interferences [16]-[19], and light scattering [20], [21] are well studied. The effect of electronic jitter as a noise source in ranging cameras is barely studied [22], [23]. Jitter is defined to be any timing movement with reference to an ideal signal. The investigation of jitter on the ToF measurements (range imaging data) is important, because it cause the errors on the measurements. Once the error sources are examined, it can be improved noise and jitter performance, and hence we can correct for these effects on range imaging data.
Jitter and/or drift between the modulation signals and the camera shutter is studied by Streeter et al. [22]. The variations in phase and frequency were modelled in a linear model by using an algorithm, which is used for jitter correction. The model is solved after computing the jitter parameters of the current frame with respect to the reference frame by using linear regression. The mean square error values with respect to the reference image are analysed to consider the effect of some filtering methods. They concluded that the combination of the filters Bilateral and Non-local Means perform well with their de-jitter algorithm, which depended only on the assumption that jitter affects equally all pixels in the scene. The second investigation by Seiter et al. [23], on the impact of two common types of cycle-to-cycle jitter distributions, namely, a random Gaussian distribution and a discrete jitter distribution on the performances of $\mathrm{ToF}$ measurements. They proved discrete jitter has a strong impact while the random Gaussian jitter decreases with the integration time, by analysing the standard deviation of the obtained measurements.

In the literature, we were unable to find an investigation on periodic jitter in ToF range imaging cameras. It is very challenging task to completely remove the sources of noise and jitter in range imaging applications, because it is more difficult to examine the camera sensor inside. We focus on jitter in the camera light source which is sensible since the light source signal is relatively straight forward to access. In this paper, we decompose the light signal into random jitter (RJ) and one of the deterministic jitter types, periodic jitter $(\mathrm{PJ})$ in a $\mathrm{ToF}$ ranging camera, namely, MESA Imaging SwissRanger 4000 [24].

The remainder of this paper is organized as follows. Section II briefly explores background theories in ToF cameras, noise and jitter components, and currently available jitter compensation techniques in relevant applications. Then, a comprehensive explanation of the proposed method with experimental set up are described in Section III. Section IV obtains the extracted jitter parameters for the camera and discuss the corresponding results. Finally, conclusion is drawn in Section V.

\section{The StATE OF ART}

In this section, the theory of operation on ToF range cameras is reviewed. Then, the jitter definition with the error sources of jitter and noise are briefly explored. Next, the current compensation techniques to resolve the jitter in real-time applications are investigated. 


\section{A. Theory of operation}

A ToF camera operates by illuminating the scene with a modulated light source and observing the reflected light via a shuttered pixelated sensor.

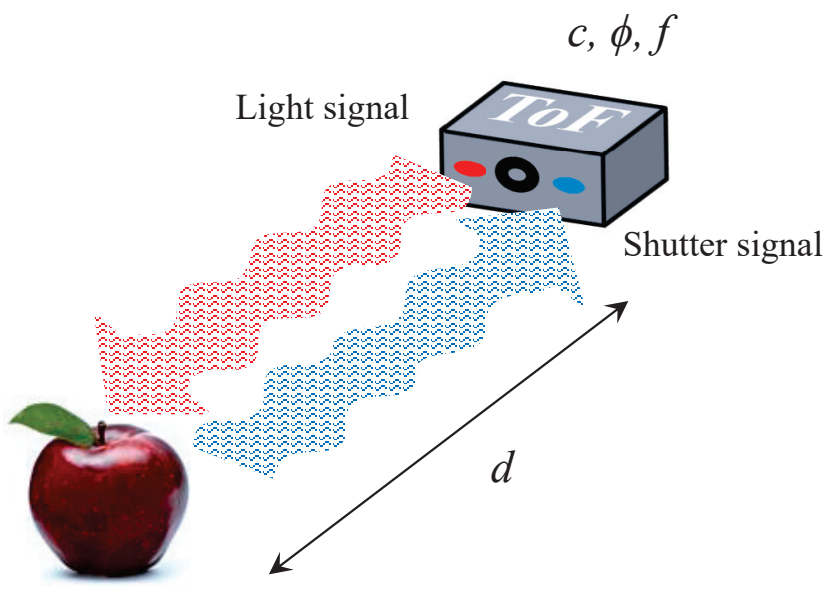

Fig. 1. A time-of-flight camera operation.

The phase shift $\phi$ due to the time-of-flight between the illumination and the reflection is measured, and from that the distance $d$ is inferred by

$$
d=\frac{c \phi}{4 \pi f}
$$

where $c$ is the speed of light and $f$ is the modulation frequency of the light source (illumination) and camera sensor. To see how to make a measurement of $\phi$ (hence $d$ ), let the emitted light source be

$$
s(t)=\sin (2 \pi f t),
$$

which is also the signal used to is electronically shutter the sensor. The reflected light signal (i.e. same light signal but phase shifted by $\theta_{k}$ for the $k$ th phase acquisition) is given by

$$
r(t)=A \sin \left(2 \pi f t+\phi+\theta_{k}\right)+B
$$

where $A$ is the amplitude of the reflected light dependent on the scene's reflectivity and the sensor's sensitivity, $B$ is the offset due to the background illumination and $\phi$ is the phase shift directly related to the scene distance. In practice, the phase shift cannot be measured directly, therefore the reflected light is correlated with the emitted light, namely,

$$
C\left(\theta_{k}\right)=\frac{1}{\tau} \int_{-\tau / 2}^{\tau / 2} r(t) s\left(t+\theta_{k}\right) d t
$$

where $\tau$ is the time delay between the two signals (i.e., integration period). Substitute (2) and (3) into (4), simplifies to

$$
C\left(\theta_{k}\right)=\frac{A}{2} \cos \left(\phi+\theta_{k}\right)+B .
$$

This correlation measurement is repeated $K$ times with a phase step of $\theta_{k}=2 \pi k / K, k=0,1, \ldots, K-1$, introduced to the camera sensor or light source. Since (5) has three unknowns $(A, B$ and $\phi)$, at least three measurements are required to perform a single estimation of them. Typically four samples (i.e., $K=4$ ) of the correlation function are sequentially acquired at phase offsets as [25]

$$
I_{k}=C\left(\theta_{k}\right) \quad \text { for } \theta_{k}=k \pi / 2, k=0, \ldots, 3,
$$

giving,

$$
\begin{array}{ll}
I_{0}=\frac{A}{2} \cos \phi+B, & I_{1}=-\frac{A}{2} \sin \phi+B, \\
I_{2}=-\frac{A}{2} \cos \phi+B, & I_{3}=\frac{A}{2} \sin \phi+B .
\end{array}
$$

Thus the phase shift due to the time of flight is given by

$$
\phi=\tan ^{-1}\left(\frac{I_{3}-I_{1}}{I_{0}-I_{2}}\right) .
$$

The depth measurements (the radial distance to the scene $d$ ) is then obtained with (1).

\section{B. Jitter definition}

A measured signal can deviate from the ideal with respect to the independent axis (time) or dependent axis (amplitude). A deviation in the time is defined as jitter. The graphical representation of jitter is shown in Fig. 2.

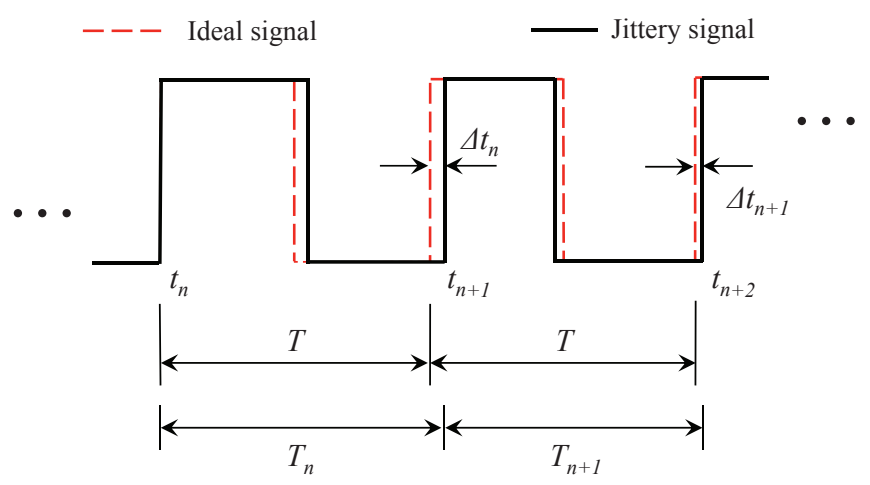

Fig. 2. Graphical representation of jitter.

The deviation of the signal $n$th timing event $t_{n}$, from its ideal occurrence in time is given by

$$
\Delta t_{n}=T_{n}+\Delta t_{n-1}-T,
$$

where $T_{n}$ is $n$th period (cycle) of the signal given by

$$
T_{n}=t_{n+1}-t_{n}
$$

and $T$ is the period of the ideal signal. Jitter can also be quantified by the time lag correlation (TLC) function $C_{\Delta t}(q)$, in terms of a time lag as [26]

$$
C_{\Delta t}(q)=\lim _{N \rightarrow \infty} \frac{1}{N} \sum_{n=1}^{N}\left(\Delta t_{n+q} \Delta t_{n}\right), \quad q \in \mathbb{Z}^{*}
$$

Here $N$ is the sample size and $q$ is the time lag number corresponding to a time lag of $q / f$. 
Jitter in a signal is often characterized as random and deterministic [27]. Random jitter (RJ) is non-deterministic jitter due to random processes. It often follows a Gaussian distribution, since most noise or jitter in an electrical circuit is caused by thermal noise, therefore, random jitter is often characterized by its root-mean-square (RMS) value. In contrast, deterministic jitter is jitter due to deterministic processes and is linearly additive with a non-Gaussian probability density function. It can be characterized by its peak or peak-topeak value. Deterministic jitter can be further categorised into several types, such as, periodic jitter (PJ), bounded correlation jitter and data dependent jitter. Since the modulation light source signal of the camera contains no data, there is no data dependent jitter in our case.

The sources of jitter in ToF cameras are generally related to the behaviour of the camera sensor, light source and transmission media of the camera. The jitter in each of these show different characteristics. Thus, it is important to identify the sources of jitter in ToF cameras [28]. Random jitter is caused by unbounded jitter sources such as white noise due to thermal noise, shot (Poisson) noise, flicker noise $(1 / f)$, and other high-order noise processes [29]. These types can occur in the camera sensor or in the light source. Power supply noise, signal modulation, crosstalk, and reflections on signals can all lead to deterministic jitter on the light or shutter modulation signals.

There are other sources of noise in ToF cameras [30], [31]. Systematic errors can be categorised by their source and include errors due to the integration time, temperature noises, built-in pixel-related noise, amplitude of reflected light and depth distortion. Several non-systematic errors in ToF cameras such as light scattering, multipath interference and motion blurs are identified in the literature. Systematic errors can be controlled by calibration methods [14], [32]-[34] while random errors in ToF can be mitigated by using signal processing techniques [18], [19], [21], [35]. Since this paper is focused on jitter, we do not further consider a noise analysis in ToF cameras.

\section{Measurement of jitter}

In general, total jitter includes both deterministic and random jitter. Here we review methods to distinguish and quantify jitter types in signals. A time-domain jitter separation method was introduced by Li et al. [36] as a general purpose automated search and non-linear fitting algorithm for jitter. They fitted the tail parts of the jitter histogram with non-linear jitter models and calculated a bit error rate for each category of jitter. To reduce deterministic jitter, adding pre-emphasis (or de-emphasis) helps by improving the signal's high-frequency content (i.e., improves SNR). Jitter can also be calculated in the frequency domain by Fourier transform. As examples, the time lag correlation (TLC) functions and fast Fourier transform (FFT) with TLC functions have been used by Dou et al. (2007) [37] and Pang et al. (2009) [38], respectively, on simulation experiments. Both of them have used a reference clock signal for the ideal signal. In order to reduce the number of equations, Pang proposed using the FFT which reduces the calculation time. In addition, random jitter and periodic jitter have been measured in simulated noisy signals by using frequency spectrum and calculating the energy of the jitter spectrum [39]. We used Fourier analysis to obtain the corresponding ideal signal for each simulated signal. Since we have no access to the reference clock signal, we use Fourier analysis method to generate the ideal signal for the camera and perform the signal processing techniques as describe in next section.

\section{Method ANd Materials}

In this section, we propose and explain an algorithm to extract the selected jitter types in ToF cameras. Analysis with calculation of jitter parameters and the experimental setup are also described.

\section{A. Proposed algorithm}

The overall plan is shown in Algorithm 1 which highlights the main stages of the algorithm used to extract the jitter components in the ToF cameras we tested.

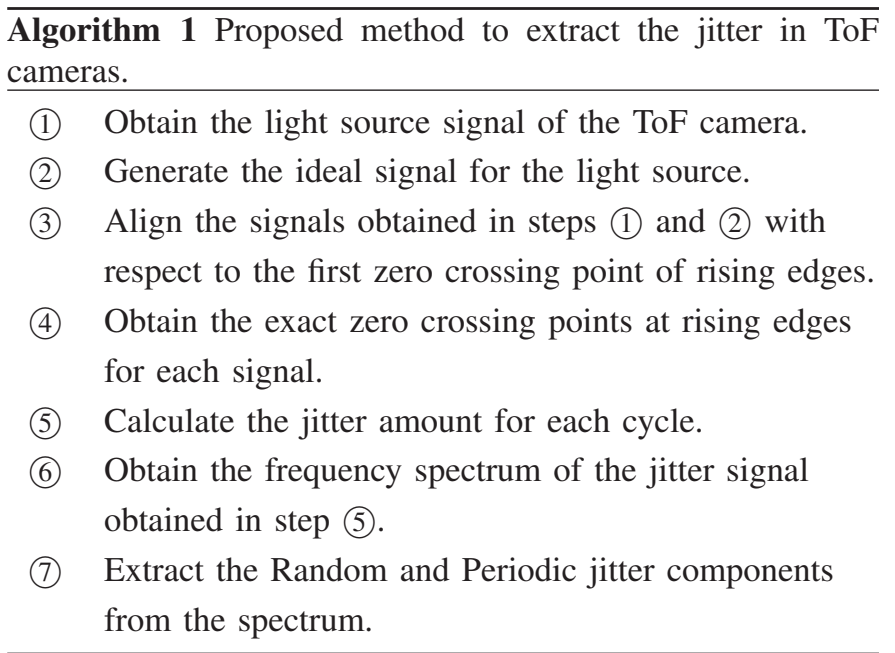

In step (1), we capture the data signal from the light source of the camera. In general, this signal consists of noisy data at the intersections of the time axis (zero crossing points). Therefore, in order to clearly identify the zero crossings of the data signal, we smooth it by using the Savitzky-Golay filtering algorithm [40] with minimal impact on the signal. Let $\mathcal{C}_{i}$ for $i=-m,-m+1, \ldots, m$ be $2 m+1$ weighting coefficients. The filtered data $y_{k}^{(s)}$ are computed by the moving average method, namely

$$
y_{k}^{(s)}=\frac{\sum_{i=-m}^{m} \mathcal{C}_{i} y_{k+i}}{\sum_{i=-m}^{m} \mathcal{C}_{i}} .
$$

The Savitzky-Golay algorithm finds the weights $C_{i}$ that are optimal in the sense that they minimize the least-squares error in fitting a polynomial to frames of noisy data. 
For step (2), we generate the corresponding sinusoidal ideal (reference) signal for the camera. For that, the relevant frequency and amplitude of the ideal signal has to be found. First, we obtain the Fourier spectrum of the smoothed data signal, find the relevant frequency at the maximum amplitude, and perform quadratic interpolation technique with its neighbours to find frequency of the ideal signal. The maximum amplitude of the smoothed data signal is used as the amplitude of the ideal signal. Next, both signals (data and ideal) are aligned with respect to the first zero crossing point of rising edges as for step (3) in our proposed algorithm. In order to find the zero crossing points, we choose the two corresponding values that sit either side of the zero crossing for each cycle. By using linear interpolation, the zero crossing points at rising edges for both signals are estimated, this being step (4).

In step (5), the jitter $\Delta t(t)$ of each rising edge of the signal is calculated at the time difference between the zero crossing points (obtained in step (4)) of the data and ideal signals of the camera. The number of cycles captured for the camera depends on the modulation frequency $f_{m}$, the number of samples $N_{s}$ in the acquired time-domain signal, and the sample rate of the acquisition $F_{s}$. This is

$$
N_{\Delta t}^{c a l}=\frac{f_{m} N_{s}}{F_{s}}
$$

Note that because the length of the signal is somewhat arbitrary there is no guarantee of complete sampled cycles of any periodic jitter present in the signal. The discrete Fourier transform assumes periodicity of the time-domain signal, which (13) violates. Therefore, we take the nearest integer value $N_{\Delta t}$ by taking the floor of (13) as

$$
N_{\Delta t}=\text { floor }\left(N_{\Delta t}^{c a l}\right)
$$

for all experiments and this is the corresponding total number of jitter samples for the camera (with respect to the acquisition setup used).

The real problem is that a $N$-point DFT assumes the signal is repeated every $N$-points throughout all time. But for arbitrary $N_{\Delta t}$ and periodic jitter frequency, this assumption is violated. This discontinuity of the signal leads to spectral leakage in the spectrum, which is the phenomenon where by the energy of a single sinusoidal signal (frequency) is spread out into neighbouring bins of the spectrum. The effects of spectral leakage can be minimized by a windowing function to smoothly attenuate both ends of the data towards zero. We use the Hann (sometimes referred as Hanning) window which is more sophisticated for random and periodic analysis for Fourier spectra [41]. The Hann window [42] is

$$
w_{H}(n)=\sin ^{2}\left(\frac{\pi n}{N-1}\right), \quad n=0,1, \ldots, N-1 .
$$

Because the signal is windowed, less energy ends up in the spectrum, and this must be taken account of. The resultant amplitudes and energies from the spectrum must be multiplied by a correction factor. The coherent gain of a window is defined as the sum of the coefficients of the window function over the interval [43]. Thus, the amplitude correction factor for the Hann window $\mathrm{A}_{H}^{c f}$, is obtained by taking the reciprocal of the coherent gain, namely

$$
\mathrm{A}_{H}^{c f}=\left(\int_{0}^{1} w_{H}(n t) \mathrm{d} t\right)^{-1}=2.0 .
$$

The incoherent gain is defined as the root of the sum of squares of the coefficients of the window function over the interval [43]. The energy correction factor for the Hann window $\mathrm{E}_{H}^{c f}$, is obtained by the reciprocal of the incoherent gain as

$$
\mathrm{E}_{H}^{c f}=\left(\sqrt{\int_{0}^{1}\left(w_{H}(n t)\right)^{2} \mathrm{~d} t}\right)^{-1}=\sqrt{8 / 3} .
$$

Then, each spectral line of the windowed frequency spectrum are multiplied by the above corresponding fixed factor. Finally, in the last step (7), the corresponding jitter parameters for the camera are extracted as describe in Section III-B.

\section{B. Jitter analysis}

Let the ideal sinusoidal signal of frequency $f$ and amplitude $A$ be

$$
i(t)=A \sin (2 \pi f t) .
$$

It is assumed both the phase shift and background offset of the signal are zero. The signal with jitter is

$$
j(t)=A \sin \left(2 \pi f\left(t+\Delta t^{R J}(t)+\Delta t^{P J}(t)\right)\right)
$$

where $\Delta t^{R J}(t)$ and $\Delta t^{P J}(t)$ are the random jitter $(\mathrm{RJ})$ and periodic jitter $(\mathrm{PJ})$, respectively. Once again, the phase shift and background offset of the signal are assumed zero. The RJ can be characterised with its RMS $\sigma_{R J}$ as

$$
\Delta t^{R J}=\alpha \sigma_{R J}, \quad \alpha \in \mathbb{R}
$$

where $\alpha$ is a Gaussian random variable.

Periodic jitter is defined as the repetition of the jitter at a certain period in a signal. In a signal, there can be multiple PJs of various frequencies and these follow the superposition rule, thus [44]

$$
\Delta t^{P J}=\sum_{p=1}^{P} A_{P J}^{(p)} \cos \left(2 \pi f_{P J}^{(p)} t\right), \quad P \in \mathbb{Z}^{+}
$$

where $A_{P J}^{(p)}$ and $f_{P J}^{(p)}$ are the amplitude and frequency of the $p$ th component of the PJ, respectively. Then, from (20) and (21), the total jitter $\Delta t(t)$ can be expressed as

$$
\Delta t(t)=\alpha \sigma_{R J}+\sum_{p=1}^{P} A_{P J}^{(p)} \cos \left(2 \pi f_{P J}^{(p)} t\right) .
$$

In the case of both RJ and PJ present, one has to find the PJ first by discrete Fourier transform (DFT). The DFT of the total jitter signal is

$$
\mathcal{F}_{k}=\sum_{n=0}^{N-1} \Delta t_{n}(t) e^{-i \frac{2 \pi n k}{N}}, \quad k=0,1, \ldots, N-1
$$


where $\mathcal{F}_{k}$ is the DFT of the $k$ th sample of the jitter signal. From the spectrum, we can easily identify the frequencies $f_{P J}^{(p)}$ of each component of the periodic jitter by peaks in the spectrum. Other jitter parameters $A_{P J}^{(p)}$ of the PJ, and $\sigma_{R J}^{2}$ for RJ can be computed by measuring the energy of the corresponding portions in the spectrum.

The total energy in the spectrum is obtained by [45]

$$
\mathrm{E}(\Delta t)=\sum_{k=0}^{N-1}\left|\mathcal{F}_{k}\right|^{2}
$$

then, the amplitude of the total jitter of the signal is given by

$$
\Delta t=\sqrt{2 \mathrm{E}(\Delta t)}
$$

where the factor $\sqrt{2}$ is the conversion of the RMS of a sinusoid to its amplitude. The corresponding energy of the $\mathrm{PJ}$ is in its peak only, thus

$$
\mathrm{E}\left(\Delta t^{P J}\right)=\sum_{p=1}^{P} \sum_{k=k_{l}^{(p)}}^{k_{u}^{(p)}}\left(\left|\mathcal{F}_{k}^{(p)}\right|^{2}-\left|\breve{\mathcal{F}}_{k}^{(p)}\right|^{2}\right),
$$

where $\mathcal{F}_{k}^{(p)}$ is the DFT of the $k$ th sample of the $p$ th component of the PJ and $\breve{\mathcal{F}}_{k}^{(p)}$ is the interpolated level of the RJ across that part of the spectrum that $p$ th component of the PJ contributes to. This part needs to be subtracted from the corresponding component of the PJ peak to be able to estimate the energy of the PJ. Terms $k_{l}^{(p)}$ and $k_{u}^{(p)}$ are the corresponding left and right indices of the interpolated level of the spectrum, respectively. Then, the amplitude of the PJ is

$$
A_{P J}=\sqrt{2 \mathrm{E}\left(\Delta t^{P J}\right)} .
$$

Next, the energy corresponding to the RJ is

$$
\mathrm{E}\left(\Delta t^{R J}\right)=\mathrm{E}(\Delta t)-\mathrm{E}\left(\Delta t^{P^{J}}\right)-\mathrm{E}(\text { none PJ) }
$$

where 'E(none PJ)' is the corresponding energy of the other spikes of the spectrum. Thus, the RMS of the RJ is

$$
\sigma_{R J}=\sqrt{\mathrm{E}\left(\Delta t^{R J}\right)} .
$$

\section{Experimental setup}

We captured the light source signal of the depth camera, MESA SR4000 (with modulation frequency $30 \mathrm{MHz}$ ) by using a Silicon biased photodetector, DET025A with two coaxial cables as 'short' $(1.070 \pm 0.005 \mathrm{~m})$ and 'long' (1.790 \pm $0.005 \mathrm{~m}$ ) measured from tip to tip of the BNC connectors. The MESA SR4000 uses infrared LEDs for its illumination source. For the control we generated $30 \mathrm{MHz}$ sine waves (corresponding to the modulation frequency of the camera) with a signal generator (HP Agilent 8648B) and measured with the oscilloscope. All data signals are measured by a real-time digital oscilloscope (HP Infiniium 54846B). The experimental setup and the relevant parameters are shown in Fig. 3 and Table I, respectively.

We calculated the number of jitter samples $N_{\Delta t}^{c a l}$ for the frequency of $30 \mathrm{MHz}$ by using (13) with the oscilloscope

\begin{tabular}{|c|c|c|c|c|}
\hline \multirow{2}{*}{ Device } & \multirow{2}{*}{$\begin{array}{c}\text { Coaxial } \\
\text { cable }\end{array}$} & \multicolumn{2}{|c|}{ No. of cycles } & \multirow{2}{*}{ EXP. \# } \\
\hline & & $N_{\Delta t}^{c a l}$ & $N_{\Delta t}$ & \\
\hline \multirow{2}{*}{$\begin{array}{l}\text { SR4000 with } \\
\text { photodetector }\end{array}$} & Short & \multirow{4}{*}{245.76} & \multirow{4}{*}{245} & EXP. \#1-ToF \\
\hline & Long & & & EXP. \#2-ToF \\
\hline \multirow{2}{*}{$\begin{array}{l}\text { Signal Generator } \\
\text { (30 MHz signal) }\end{array}$} & Short & & & EXP. \#1-SG \\
\hline & Long & & & EXP. \#2-SG \\
\hline
\end{tabular}
parameters $N_{s}=2^{16}$ and $F_{s}=8 \mathrm{GSa} / \mathrm{s}$. The $N_{\Delta t}^{c a l}$ and

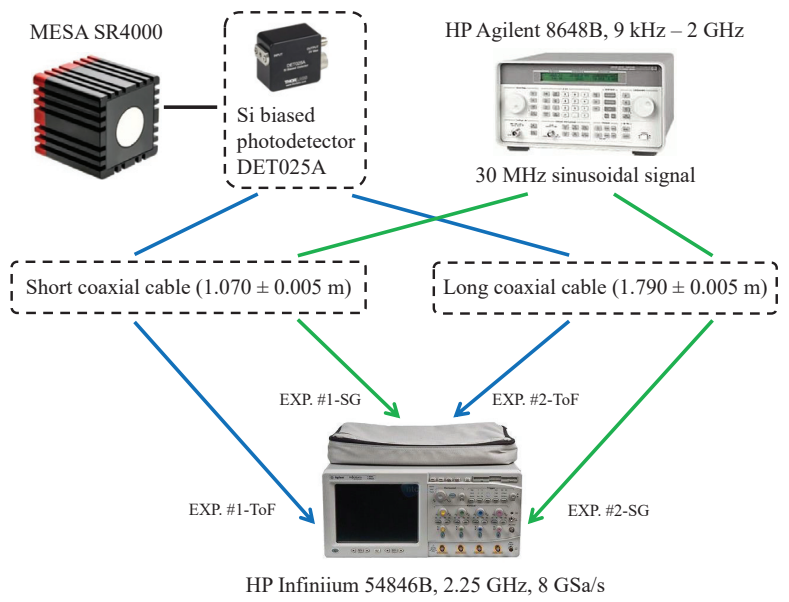

Fig. 3. Experimental setup.

TABLE I

PARAMETERS OF THE EXPERIMENTAL SETUP

the nearest integer value $N_{\Delta t}$ are tabulated in columns three and four, respectively, in Table I. In order to reduce the uncertainty of the result, we captured 1000 acquisitions for each experimental setup and obtained the total jitter spectrum for each acquisition by using the steps (1) to (6) in proposed algorithm.

\section{RESUlTS AND Discussion}

Figure 4 shows the results for the corresponding steps (1) to (3) of the proposed algorithm for a single acquisition for both the camera and the signal generator. The left and right sub-figures correspond to EXP. ToF (i.e., for SR4000) and EXP. SG (i.e., for $30 \mathrm{MHz}$ signal), respectively. By comparison the two sub-figures in Fig. 4(a), it is clear that the noise in the data signal (light source) of SR4000 (left of Fig. 4(a)) is relatively higher than the same in $30 \mathrm{MHz}$ signal (right of Fig. 4(a)). The smoothed data signal obtained by the SavitzkyGolay algorithm shows minimal impact on the zero crossings at the time axis (see Fig. 4(b)). Then, the data signal and the corresponding ideal signal is aligned with respect to the first zero crossing point of the rising edge as shown in Fig. 4(c). After applying steps (4) to (6), the spectrum of the total jitter for each experiment, is obtained. The total jitter spectrum for each experiment (SR4000 and $30 \mathrm{MHz}$ signal) is illustrated in Fig. 5. In addition, jitter components and the interpolated level between periodic and random jitter for each signal is marked in the figure. 

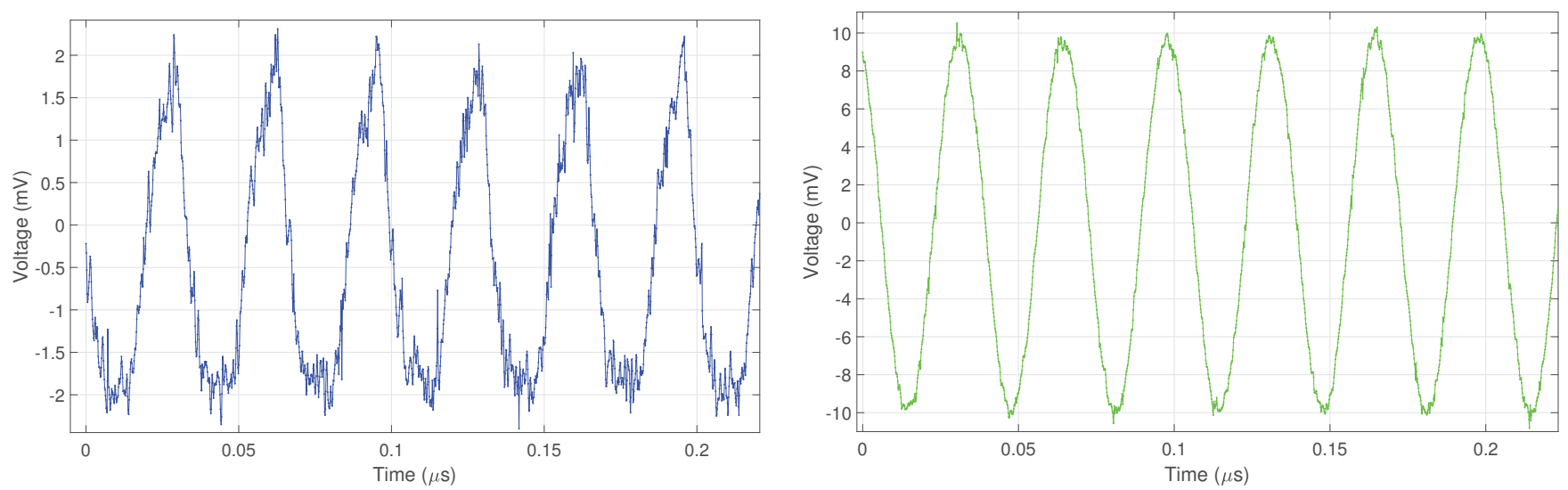

(a)
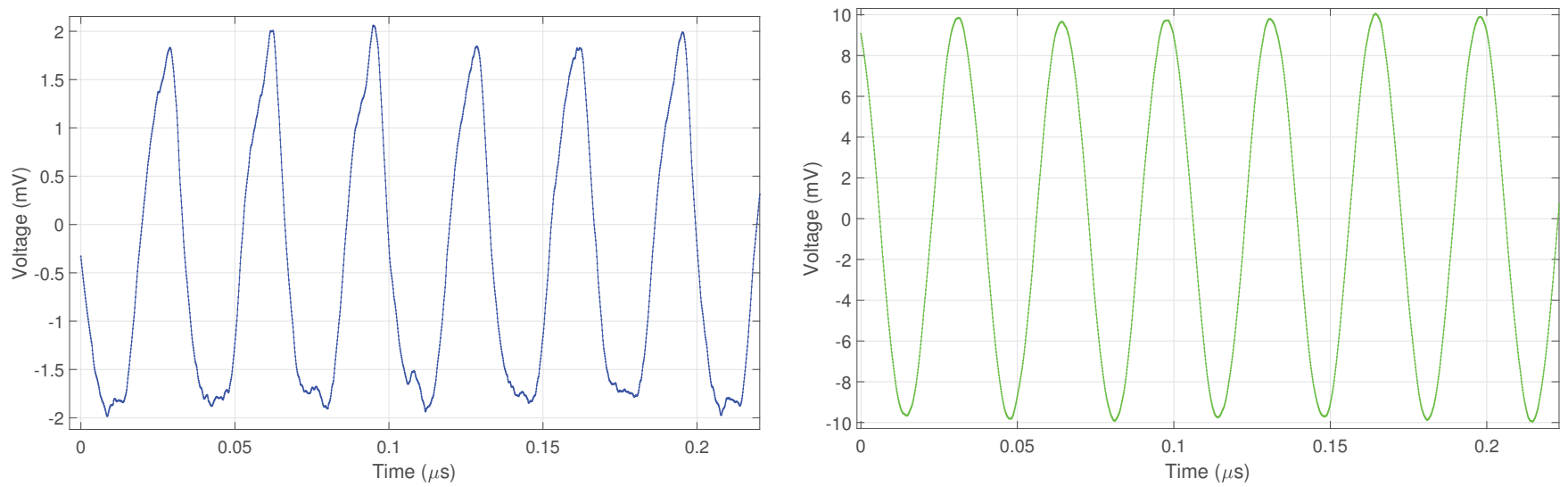

(b)
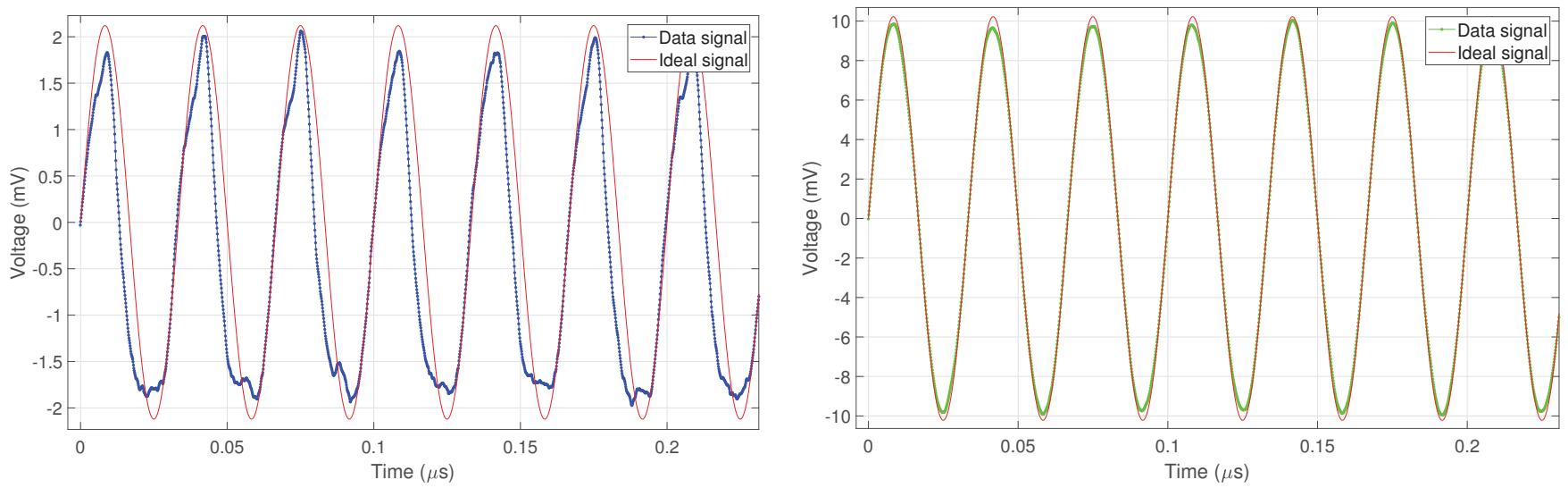

(c)

Fig. 4. Steps (1) to (3) of proposed method in Algorithm 1: (a) step (1) - light source (data) signal, (b) smoothed data signal (using Savitzky-Golay algorithm), and (c) step (3) - aligned the data and ideal signals, for the single acquisition of SR4000 (left) and $30 \mathrm{MHz}$ signal (right). 


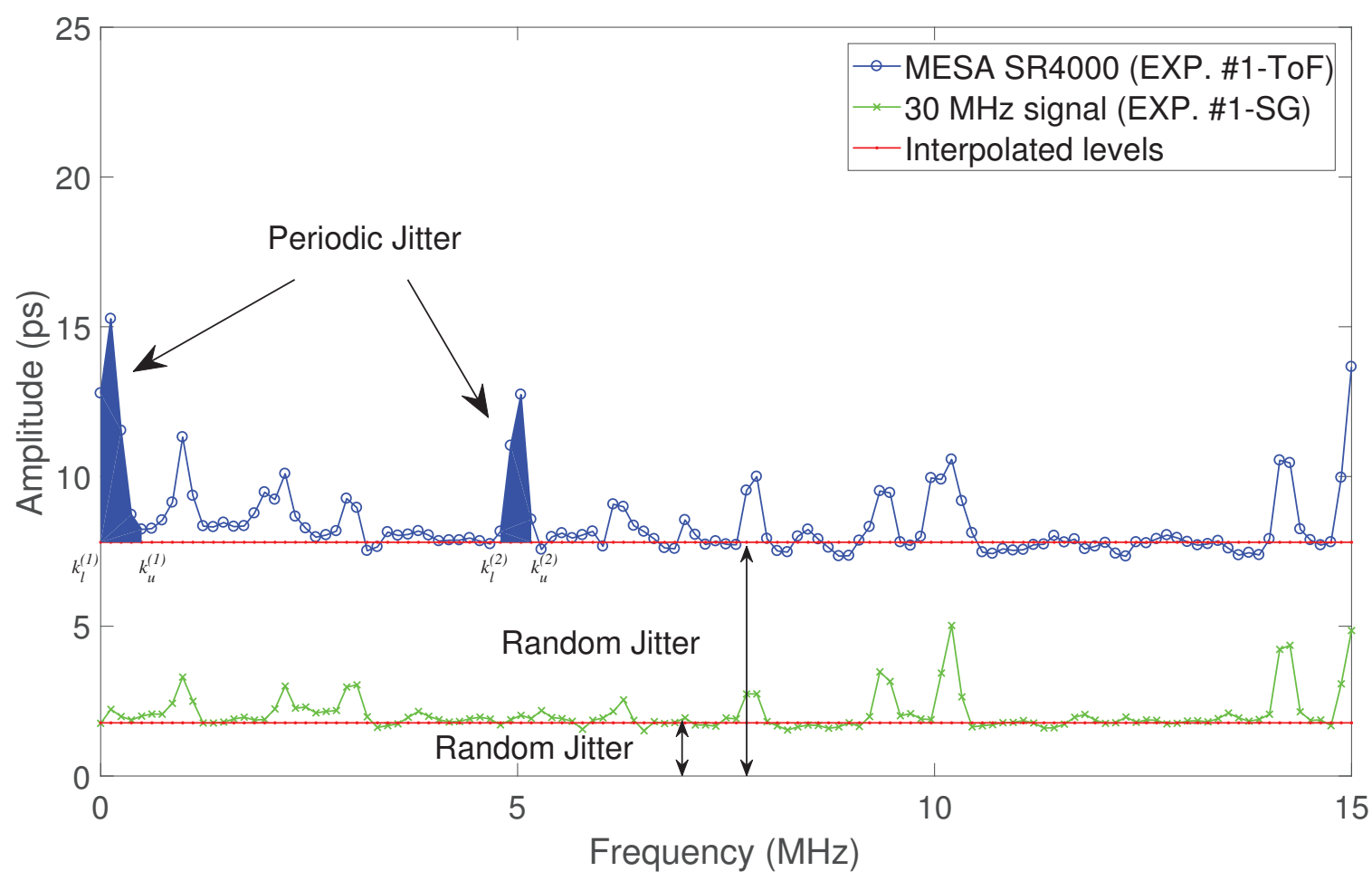

(a)

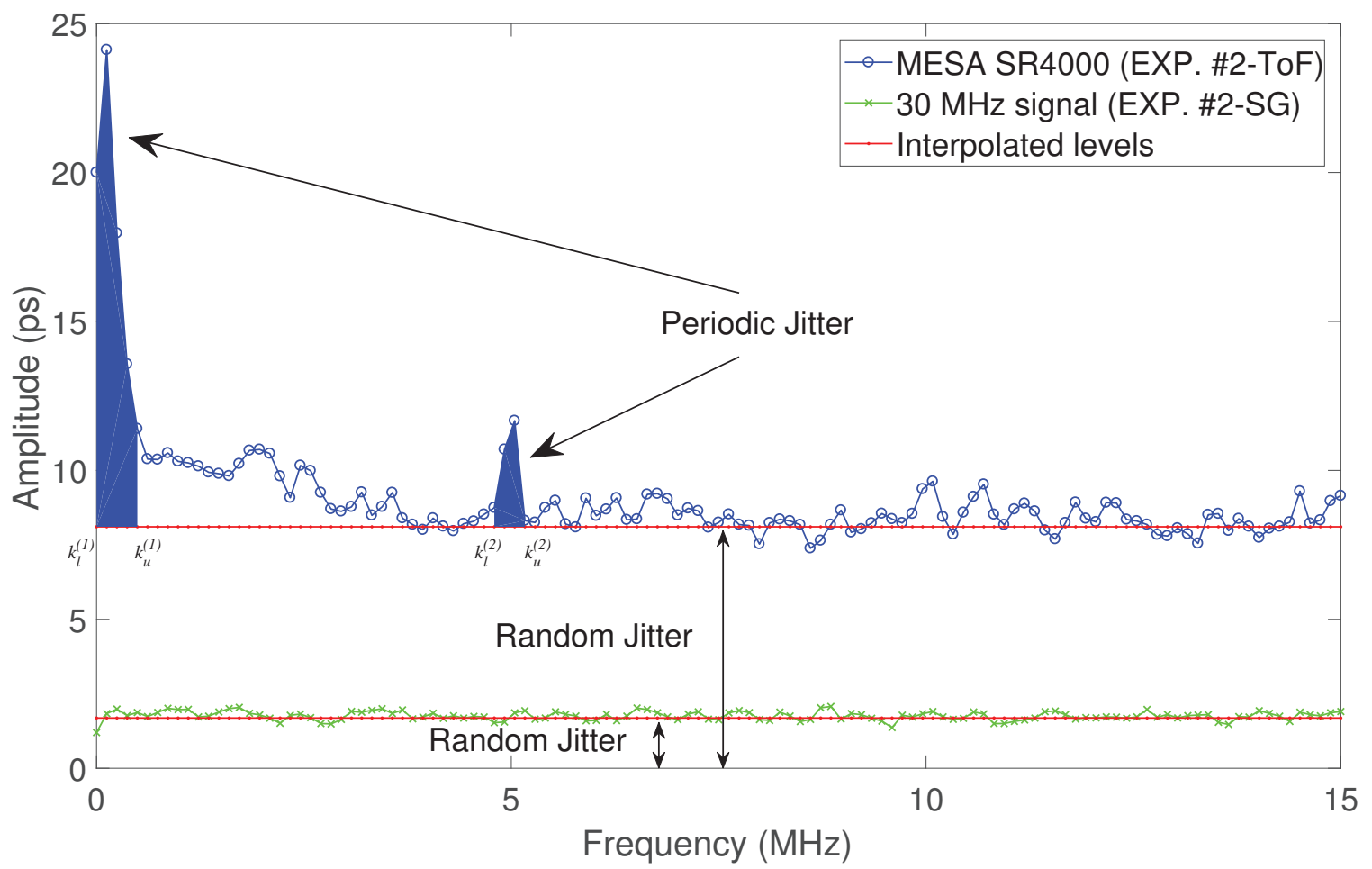

(b)

Fig. 5. Representation of jitter components for the experiments (a) EXP. \#1 and (b) EXP. \#2. 


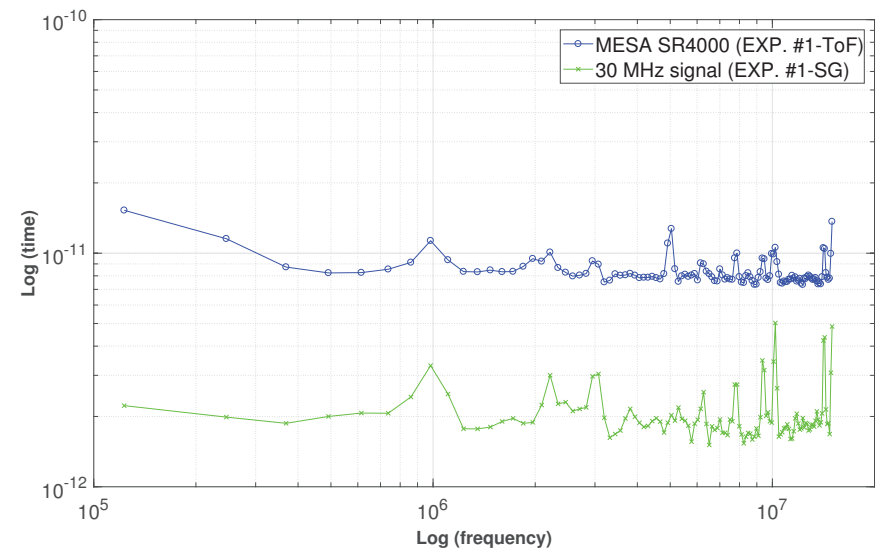

(a)

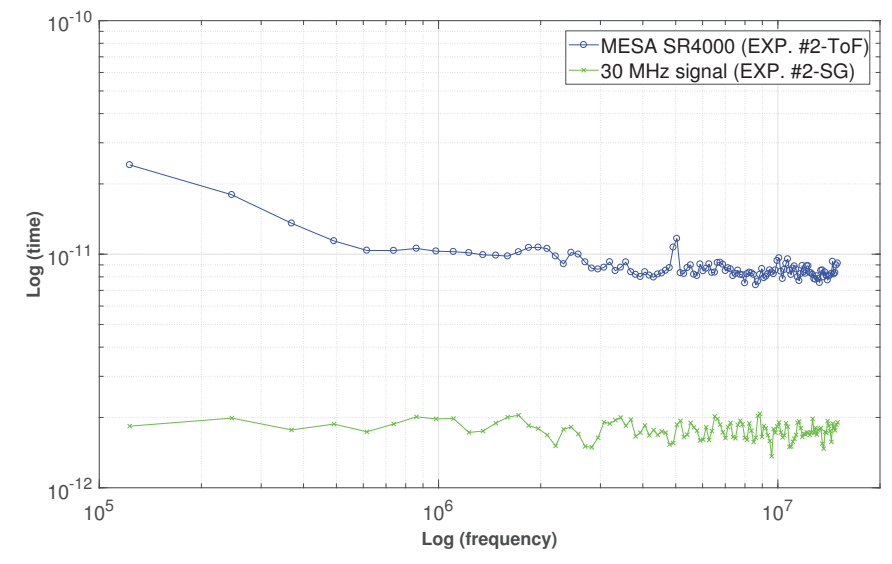

(b)

Fig. 6. Log-log scale with fitted curves for the spectra in Fig. 5 for (a) EXP. \#1 and (b) EXP. \#2, as given in Table I.

By comparing the two jitter signals in each corresponding experiment, it is clear that there are two new high peaks at around 0 and $5 \mathrm{MHz}$ on the jitter signal of SR4000 (i.e., EXP. \#1-ToF and EXP. \#2-ToF) which are not present in the jitter signal of the signal generator (i.e., EXP. \#1-SG and EXP. \#2$\mathrm{SG})$. There is also a broad and flat spectrum being the random jitter at the levels of approximately 8 and 2 ps for SR4000 and $30 \mathrm{MHz}$ signal, respectively. Furthermore, there is peaking noise, particularly in the $1.07 \mathrm{~m}$ cable experiments. Due to the fact that they are reduced by using the $1.79 \mathrm{~m}$ cable we believe that these are due such as the cables and connectors.

The corresponding log-log scales for total jitter is illustrated in Fig. 6. By using the spectra of the total jitter, it is straightforward to obtain the corresponding frequencies of the periodic jitter $f_{P J}^{(p)}$ in SR4000. It is given as 0.12 and $5.04 \mathrm{MHz}$ (see Fig. 5) for both cases. Other jitter parameters are calculated by considering the energy of the spectrum as described in Section III-B. Note that, all the energy calculations are compensated with the energy correction factor using (17) before calculate the jitter parameters. Then, the parameters $A_{P J}^{(p)}$ and $\sigma_{R J}$ are calculated by using (27) and (29), respectively. The calculated jitter parameters are tabulated in Table II. The tabulated results are obtained by considering the full spectrum of the jitter signal for each experiment. For each experiment, the corresponding frequency of each ideal signal (a pure sine wave without jitter, extracted from the data) is included in column three. The standard deviation of the frequencies are also provided as a measure of precision. The signal generator and ToF camera frequencies are within one standard deviation each other. Also, the standard deviations are small compared to the ideal signal, with precision to four and five significant digits for the ToF light source and signal generator, respectively. Therefore, we have measured the frequency to sufficient precision for our purposes.

As usual there is no periodic jitter in the signal generator 30 $\mathrm{MHz}$ signal in both cases. The random jitter is 35 and $34 \mathrm{ps.}$ Furthermore, the curvature at low frequencies of the total jitter
TABLE II

JITTER PARAMETERS FROM THE EXPERIMENTS

\begin{tabular}{|c|c|c|c|c|}
\hline \multicolumn{2}{|c|}{ EXP. } & Frequency of ideal & $\begin{array}{c}\text { Periodic Jitter } \\
\text { \# }\end{array}$ & RJ \\
\hline \multirow{2}{*}{1} & setup & signal $(\mathrm{MHz})$ & {$\left[f_{P J}^{(p)}(\mathrm{MHz}), A_{P J}^{(p)}(\mathrm{ps})\right]$} & $\sigma_{R J}(\mathrm{ps})$ \\
\cline { 2 - 4 } & ToF & $30.006 \pm 0.0012$ & {$[0.12,61] \&[5.04,34]$} & 160 \\
& SG & $30.006 \pm 0.0006$ & null & 35 \\
\hline \multirow{2}{*}{2} & ToF & $30.005 \pm 0.0011$ & {$[0.12,103] \&[5.04,30]$} & \multirow{2}{*}{168} \\
\cline { 2 - 4 } & SG & $30.006 \pm 0.0006$ & null & 34 \\
\hline
\end{tabular}

for SR4000 is higher than at the rest of the frequencies (see Fig. 5) in each case. We expect this may be due to the flicker noise $(1 / f)$ in the camera. This trends is more larger in EXP. \#2-ToF than in EXP. \#1-ToF since in the later case it presents a larger periodic jitter component at the low frequency. Then this may significantly leaked the energy of the signal to adjacent bins of the spectrum. As usually, we believe the measurements from the oscilloscope have a good accuracy. The minimum RMS value of jitter that can be measured by the oscilloscope is 8 ps.

\section{CONClusion}

In this paper, we propose a method for the extraction of periodic and random jitter in depth cameras and experimented with MESA SR4000. This method can be used to real time jitter investigation for most of the ToF range imaging cameras since the light sources of these cameras are directly accessible. The most important and the advantage of this method is, it is not required a reference or sampling clock signal to trigger the time measurement unit. We used the Fourier analysis and the maximum amplitude of among the multiple data set as for the corresponding frequency and amplitude, respectively, for the ideal signal of the light source of the camera and for the 30 $\mathrm{MHz}$ sine wave of the signal generator, separately. We found the periodic jitter of two frequencies at 0.12 and $5.04 \mathrm{MHz}$ 
with the amounts of $82 \pm 21$ and $32 \pm 2$ ps, respectively, on the light signal of the camera. In addition, SR4000 has relatively higher random jitter $(164 \pm 4 \mathrm{ps})$ than periodic jitter in the range measurements. In future work, we plan to investigate how these two types of jitter in depth cameras effect the range measurements. This helpful to increase the accuracy and precision of the range measurements from depth cameras used in industrial applications.

\section{REFERENCES}

[1] F. Blais, "Review of 20 years of range sensor development," Journal of Electronic Imaging, vol. 13, no. 1, 2004.

[2] L. Li, "Time-of-flight camera - an introduction," Texas Instruments Technical White Paper, Dallas, Texas 75265, U.S.A., Tech. Rep., 2014

[3] M. Laukkanen, "Performance evaluation of time-of-flight depth cameras," Master, Aalto university, 2015.

[4] J. S. Karp, S. Surti, M. E. Daube-Witherspoon, and G. Muehllehner, "The benefit of time-of-flight in pet imaging: Experimental and clinical results," Journal of nuclear medicine: official publication, Society of Nuclear Medicine, vol. 49, no. 3, p. 462, 2008.

[5] S. Soutschek, J. Penne, J. Hornegger, and J. Kornhuber, "3-d gesturebased scene navigation in medical imaging applications using time-offlight cameras," 2008.

[6] A. Kolb, E. Barth, R. Koch, and R. Larsen, "Time-of-flight cameras in computer graphics," in Computer Graphics Forum, vol. 29, no. 1. Wiley Online Library, 2010, pp. 141-159.

[7] S. May, B. Werner, H. Surmann, and K. Pervolz, "3d time-of-flight cameras for mobile robotics," in Intelligent Robots and Systems, 2006 IEEE/RSJ International Conference on. Ieee, 2006, pp. 790-795.

[8] T. Schamm, M. Strand, T. Gumpp, R. Kohlhaas, J. M. Zollner, and R. Dillmann, "Vision and tof-based driving assistance for a personal transporter," in Advanced Robotics, 2009. ICAR 2009. International Conference on. IEEE, 2009, pp. 1-6.

[9] P. Henry, M. Krainin, E. Herbst, X. Ren, and D. Fox, "Rgb-d mapping: Using depth cameras for dense $3 \mathrm{~d}$ modeling of indoor environments," in 12th International Symposium on Experimental Robotics (ISER. Citeseer, 2010.

[10] X. Liu and K. Fujimura, "Hand gesture recognition using depth data," in 6th International Conference on Automatic Face and Gesture Recognition. IEEE, 2004, p. 529.

[11] J. Tong, J. Zhou, L. Liu, Z. Pan, and H. Yan, "Scanning 3d full human bodies using kinects," IEEE transactions on visualization and computer graphics, vol. 18, no. 4, pp. 643-650, 2012.

[12] X.-L. Jin and S.-Q. Zeng, "Design of 3D TOF camera system based on CW modulation technique," in 2016 13th IEEE International Conference on Solid-State and Integrated Circuit Technology (ICSICT), Oct 2016, pp. 373-375.

[13] C. D. M. Pietro Zanuttigh, Giulio Marin, Time-of-Flight and Structured Light Depth Cameras: Technology and Applications, 1st ed. Springer International Publishing, 2016.

[14] T. Kahlmann, F. Remondino, and H. Ingensand, "Calibration for increased accuracy of the range imaging camera Swissranger $T M$," in Image Engineering and Vision Metrology, ser. 3, vol. 36, 2006, pp. 136141.

[15] L. Streeter and A. A. Dorrington, "Simple harmonic error cancellation in time of flight range imaging," Optics Letters, vol. 40, no. 22, pp. 5391-5394, 2015.

[16] J. P. Godbaz, M. J. Cree, and A. A. Dorrington, "Understanding and ameliorating non-linear phase and amplitude responses in AMCW lidar," Remote Sensing, vol. 4, no. 1, pp. 21-42, 2011.

[17] D. Freedman, Y. Smolin, E. Krupka, I. Leichter, and M. Schmidt, "Sra: Fast removal of general multipath for tof sensors," in Computer VisionECCV 2014. Springer, 2014, pp. 234-249.

[18] D. Jiménez, D. Pizarro, M. Mazo, and S. Palazuelos, "Modeling and correction of multipath interference in time of flight cameras," Image and Vision Computing, vol. 32, no. 1, pp. 1-13, 2014.

[19] R. Whyte, L. V. Streeter, M. J. Cree, and A. A. Dorrington, "Resolving multiple propagation paths in time of flight range cameras using direct and global separation methods," Optical Engineering, vol. 54, no. 11, pp. $1-9,2015$
[20] J. Mure-Dubois and H. Hügli, "Real-time scattering compensation for time-of-flight camera," in Proceedings of the ICVS Workshop on Camera Calibration Methods for Computer Vision Systems, 2007.

[21] H. Schäfer, F. Lenzen, and C. S. Garbe, "Model based scattering correction in time-of-flight cameras," Optics express, vol. 22, no. 24, pp. 29835-29846, 2014.

[22] L. Streeter, M. J. Cree, A. Dorrington et al., "A strategy for the correction of effects of jitter in AMCW lidar images," in Image and Vision Computing New Zealand (IVCNZ), 2013 28th International Conference of. IEEE, 2013, pp. 500-505.

[23] J. Seiter, M. Hofbauer, M. Davidovic, and H. Zimmermann, "Investigation of the distance error induced by cycle-to-cycle jitter in a correlating time-of-flight distance measurement system," Optical Engineering, vol. 53, no. 7, pp. 073 104-073 104, 2014.

[24] Industrial products - swissranger. http://hptg.com/industrial/.

[25] R. Lange and P. Seitz, "Solid-state time-of-flight range camera," IEEE Journal of Quantum Electronics, vol. 37, no. 3, pp. 390-397, Mar 2001.

[26] F. Herzel and B. Razavi, "A study of oscillator jitter due to supply and substrate noise," IEEE Transactions on Circuits and Systems II: Analog and Digital Signal Processing, vol. 46, no. 1, pp. 56-62, Jan 1999.

[27] Primer, "Understanding and characterizing timing jitter," Tektronix, Beaverton, OR 97077, United States, Tech. Rep., 2012.

[28] J. Hancock and A. Technologies, "Jitter fundamentals," High Frequency Electronics, pp. 44-50, April 2004.

[29] H. Rapp, M. Frank, F. Hamprecht, and B. Jhne, "A theoretical and experimental investigation of the systematic errors and statistical uncertainties of time-of-flight-cameras," Int. J. Inte. Sys. Tech. and Appl., vol. 5, no. 3/4, 2008.

[30] D. Falie and V. Buzuloiu, "Noise characteristics of 3D time-of-flight cameras," in 2007 International Symposium on Signals, Circuits and Systems, vol. 1, July 2007, pp. 1-4.

[31] S. Foix, G. Alenya, and C. Torras, "Lock-in Time-of-Flight (ToF) Cameras: A Survey," IEEE Sensors Journal, vol. 11, no. 9, pp. 19171926, Sept 2011.

[32] S. Oprisescu, D. Falie, M. Ciuc, and V. Buzuloiu, "Measurements with tof cameras and their necessary corrections," in Signals, Circuits and Systems, 2007. ISSCS 2007. Int. Symposium on, vol. 1, July 2007.

[33] Y. M. Kim, D. Chan, C. Theobalt, and S. Thrun, "Design and calibration of a multi-view TOF sensor fusion system," in Computer Vision and Pattern Recognition Workshops, 2008. CVPRW '08. IEEE Computer Society Conference on, June 2008, pp. 1-7.

[34] S. Hussmann, F. Knoll, and T. Edeler, "Modulation method including noise model for minimizing the wiggling error of tof cameras," IEEE Transactions on Instrumentation and Measurement, vol. 63, no. 5, pp. 1127-1136, May 2014.

[35] S. Jamtsho and D. D. Lichti, "Modelling scattering distortion in 3d range camera," International Archives of Photogrammetry, Remote Sensing and Spatial Information Sciences, vol. 38, no. 5, pp. 299-304, 2010.

[36] M. P. Li, J. Wilstrup, R. Jessen, and D. Petrich, "A new method for jitter decomposition through its distribution tail fitting," in International Test Conference 1999. Proceedings (IEEE Cat. No.99CH37034), 1999, pp. 788-794.

[37] Q. Dou and J. A. Abraham, "Jitter decomposition by time lag correlation," in 7th Inter. Symp. on Quality Electronic Design (ISQED'06), March 2006, pp. 6 pp.-530.

[38] H. Pang, J. Zhu, and W. Huang, "Jitter decomposition by fast Fourier transform and time lag correlation," in 2009 Inter. Conf. on Communications, Circuits and Systems, July 2009, pp. 365-368.

[39] G. Anthonys, M. J. Cree, and L. Streeter, "Jitter extraction in a noisy signal by fast fourier transform and time lag correlation," Applied Mechanics and Materials, vol. 884, pp. 113-121, 92018.

[40] S. Orfanidis, Introduction to Signal Processing. Prentice Hall, 1996.

[41] A. V. Oppenheim, R. W. Schafer, and J. R. Buck, Discrete-time Signal Processing (2nd Ed.). Upper Saddle River, NJ, USA: Prentice-Hall, Inc., 1999.

[42] R. B. Blackman and J. W. Tukey, "The measurement of power spectra from the point of view of communications engineering part i," The Bell System Technical Journal, vol. 37, no. 1, pp. 185-282, Jan 1958.

[43] R. G. Lyons, Understanding Digital Signal Processing, 3rd ed. Boston, MA, USA: Pearson Education, Inc., 2011.

[44] M. Li, Jitter, Noise, and Signal Integrity at High-speed, 1st ed. Upper Saddle River, NJ, USA: Prentice Hall Press, 2007.

[45] R. B. Randall, Frequency Analysis, 3rd ed. Brül \& Kjor, 1987. 